\title{
Breaking the Symmetry of Forward-Backward Light Emission with Localized and Collective Magnetoelectric Resonances in Arrays of Pyramid-Shaped Aluminum Nanoparticles
}

\author{
S. R. K. Rodriguez, ${ }^{1, *}$ F. Bernal Arango, ${ }^{2}$ T. P. Steinbusch, ${ }^{1}$ M. A. Verschuuren, ${ }^{3}$ A. F. Koenderink, ${ }^{2}$ and J. Gómez Rivas ${ }^{1,4}$ \\ ${ }^{1}$ Center for Nanophotonics, FOM Institute AMOLF, clo Philips Research Laboratories, \\ High Tech Campus 4, 5656 AE Eindhoven, The Netherlands \\ ${ }^{2}$ Center for Nanophotonics, FOM Institute AMOLF, Science Park 104, 1098 XG Amsterdam, The Netherlands \\ ${ }^{3}$ Philips Research Laboratories, High Tech Campus 4, 5656 AE Eindhoven, The Netherlands \\ ${ }^{4}$ COBRA Research Institute, Eindhoven University of Technology, P.O. Box 513, 5600 MB Eindhoven, The Netherlands
}

(Received 27 January 2014; published 12 December 2014)

\begin{abstract}
We propose aluminum nanopyramids (ANPs) as magnetoelectric optical antennas to tailor the forward versus backward luminescence spectrum. We present light extinction and emission experiments for an ANP array wherein magnetoelectric localized resonances couple to in-plane diffracted orders. This coupling leads to spectrally sharp collective resonances. Luminescent molecules drive both localized and collective resonances, and we experimentally demonstrate an unconventional forward versus backward luminescence spectrum. Through analytical calculations, we show that the magnetic, magnetoelectric, and quadrupolar moments of ANPs - which lie at the origin of the observed effects - are enhanced by their tapering and height. Full-wave simulations show that localized and delocalized magnetic surface waves, with an excitation strength depending on the plane wave direction, direct the forward versus backward emitted intensity.
\end{abstract}

DOI: 10.1103/PhysRevLett.113.247401

In a celebrated paper, Pendry and co-workers laid the theoretical foundation for enhancing magnetism at optical frequencies with metals [1]. When subwavelength features are imprinted on metals, magnetism emerges from the coupled capacitive and inductive response to electromagnetic radiation. This principle has guided great advances in the field of metamaterials: artificial structures possessing properties absent in natural materials. Initial interest in metamaterials focused on their effective medium properties [2], aimed at the realization of nontrivial phenomena such as negative refraction [3,4], subwavelength resolution [5-7], and cloaking [8-10]. Recently, interest has extended towards understanding the electric, magnetic, and magnetoelectric response of the constituent resonators [11-15]. Such an understanding has important connections to the field of optical antennas, where the constituent metamaterial resonators interface plane waves with localized light sources [16]. Thus, for metamaterials as for optical antennas, magnetoelectric resonators are pivotal for achieving full control of electromagnetic waves. Among the possibilities enabled by this control, an effect that has attracted increasing interest is the modification of forward and backward scattering in anomalous ways. This occurs for dielectric nanoparticles when electric and magnetic moments are comparable in magnitude, at the so-called Kerker condition [17-20]. Unbalanced forward and backward scattering has inspired several studies revisiting fundamental concepts in electromagnetism (e.g., the optical theorem [21]) and predicting intriguing effects such as cloaked sensors [10] and nanoscale control of electromagnetic hot spots [22]. However, the unbalanced forward and backward emission from planar light sources, e.g., thin luminescent layers,
PACS numbers: 78.67.Bf, 42.25.Fx, 73.20.Mf, 81.05.Xj

remains unexplored. Interestingly, since planar structures are often designed to emit into the "forward" direction and the backward emission leads to losses, controlling this directional emission represents an important functionality that magnetoelectric nanoantennas may address.

In this Letter we demonstrate strong spectral modifications in the forward versus backward emission from planar sources coupled to magnetoelectric metallic nanostructures. We investigate aluminum nanopyramids (ANPs) sustaining localized and collective resonances driven by luminescent molecules. Collective resonances arise in periodic arrays of metallic nanoparticles when in-plane diffracted orders-so-called Rayleigh anomalies (RAs) - couple to localized surface plasmons [23-32]. This coupling leads to hybrid plasmonicphotonic resonances known as surface lattice resonances (SLRs), with quality factors depending on the number of particles in the array [33]. SLRs have attracted interest for their tunable linewidths and dispersion $[32,34,35]$, enhanced spontaneous emission [36-40], sensing [41,42], lasing [43], and strong coupling to molecules [44-46]. While previous studies focused on the diffractive coupling of particles with a dominant electric response, here we investigate light emission from a structure supporting diffractive coupling of particles with comparable electric and magnetic response. To begin, we demonstrate how the bare resonators can be geometrically designed to sustain such a response. Next, we present experiments for a lattice of resonators with enhanced magnetic and quadrupolar response, demonstrating an unconventional forward versus backward luminescence enhancement. Finally, through full-wave simulations we link these effects to the directional excitation strength of localized and delocalized magnetic surface waves in the array. 
We choose aluminum to operate in the visible spectrum: its relatively high plasma frequency leads to blue-shifted resonances with respect to gold and silver structures with equal dimensions. To study the geometrically dependent response of individual ANPs separate from lattice and interface effects, we calculated their $11 \times 11$ superpolarizability tensor $\alpha^{S}[47-49]$ in free space $(n=1) . \alpha^{S}$ quantifies the electric dipolar, magnetic dipolar, and quadrupolar moments of an arbitrary structure. The upper left $6 \times 6$ elements of $\alpha^{S}$ describe the excitation of dipolar electric $\mathbf{p}$ and magnetic $\mathbf{m}$ moments by electric $\mathbf{E}$ and magnetic $\mathbf{H}$ driving fields. In this block, the diagonal $3 \times 3$ blocks represent purely electric and magnetic polarizability $\left(\mathbf{p}=\alpha_{E} \mathbf{E}\right.$, and $\mathbf{m}=\alpha_{H} \mathbf{H}$ ) dipoles, whereas the off-diagonal magnetoelectric coupling terms represent the excitation of electric (magnetic) dipoles by magnetic (electric) fields. The bottom five rows of $\alpha^{S}$ describe the quadrupolar moments $\mathbf{Q}$ driven by $\mathbf{E}$ and $\mathbf{H}$. The rightmost five columns represent the induced dipole moments $\mathbf{p}$ and $\mathbf{m}$ due to symmetric gradients of the field $\diamond \mathbf{E}$ [50], with the symmetric gradient operator $\diamond$ defined as $\diamond \mathbf{E}=(1 / 2)\left\{\left(\partial_{x} E_{y}+\partial_{y} E_{x}\right),\left(\partial_{x} E_{z}+\partial_{z} E_{x}\right)\right.$, $\left.\left(\partial_{y} E_{z}+\partial_{z} E_{y}\right), 2 \partial_{x} E_{x}, 2 \partial_{y} E_{y}\right\}$ when acting over a 3 -vector $\mathbf{E}$. The $5 \times 5$ lowest diagonal block is the quadrupolarizability tensor [53], representing the quadrupolar moments excited by $\diamond \mathbf{E}$. The superpolarizability is obtained by full-wave calculations for 11 linearly independent incident conditions of the object scattering response, which we project onto vector spherical harmonics [49].

Figure 1(a) shows a graphical representation of $\alpha^{S}$ for ANPs with a top size of $t=84 \mathrm{~nm}$, height $h=150 \mathrm{~nm}$, and base $b=144 \mathrm{~nm}$, in reference to Fig. 1(b). The excitation wavelength is $660 \mathrm{~nm}$. In the system of units we use (see Supplemental Material for details [50]), all elements of $\alpha^{S}$ can be compared quantitatively, i.e., as yielding identical scattering power at identical value. The ANPs in Fig. 1(a) possess a strong magnetoelectric cross-coupling polarizability $\alpha_{C}$ (tensor elements [4,2], [2,4], [5,1], and [1,5]). Comparing this to the electric polarizability, we find $\left|\alpha_{E}\right| /\left|\alpha_{C}\right|=13$. This strong magnetoelectric response of ANPs at visible frequencies resembles the response of split ring resonators at infrared frequencies [48,54]. Figure 1(a) further shows that $x$ or $y$ polarized plane waves, without a strong gradient, directly induce quadrupoles in the $x z$ and $y z$ planes. The magnetic dipoles along $x$ and $y$ and the quadrupoles in the $y z$ and $x z$ planes, respectively, are related through the rotational symmetry of the structure.

In Figs. 1(c) and 1(d) we study the influence of tapering and height on the most significant elements of $\alpha^{S}$ for $x$ polarized illumination [indicated in Fig. 1(b)]. We divide $\alpha^{S}$ by the volume of each structure to make a fair comparison [50]. $\alpha^{S}$ is evaluated at the electric dipolar resonance, which varies from $660 \mathrm{~nm}$ for different structures [50]. To begin, we set $b=144 \mathrm{~nm}$. This enhances $\mathbf{m}$ and $\mathbf{Q}$ with respect to $\mathbf{p}$ (in contrast to smaller structures) and matches the experiments shown ahead. For $h=150 \mathrm{~nm}$ and varying $t$, Fig. 1(c) shows that the magnetoelectric $\left(\alpha_{m y}^{E x}\right)$ and quadrupolar $\left(\alpha_{Q x z}^{E x}\right)$ responses increase monotonically by 3 orders of magnitude with tapering. We attribute this to the broken
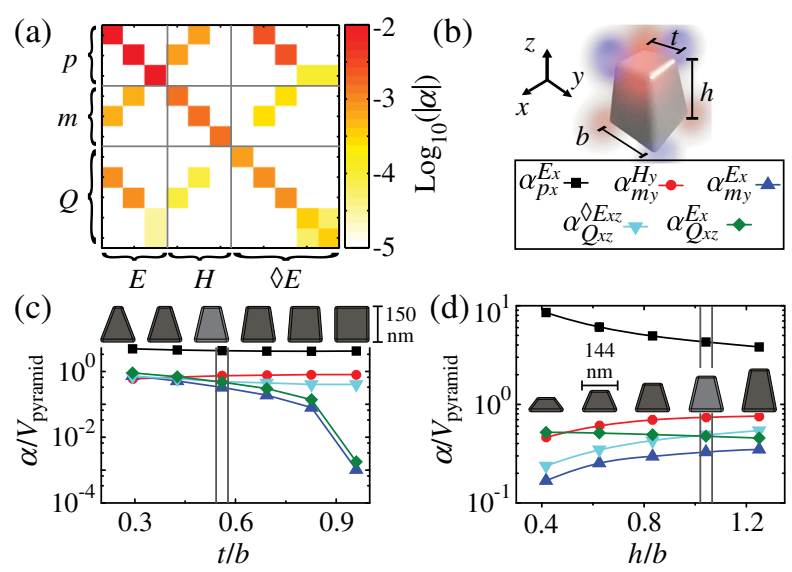

FIG. 1 (color online). (a) Superpolarizability tensor $\alpha^{S}$ of an aluminum pyramid with $t=84 \mathrm{~nm}, h=150 \mathrm{~nm}$ and $b=144 \mathrm{~nm}$, at the electric dipolar resonance wavelength. (b) Schematic of the structure, and legend of Figs. (c),(d). (c) and (d) display the most significant elements of $\alpha^{S}$ as a function of the tapering and the height of the pyramids, respectively.

mirror symmetry about the ANPs midheight, as it occurs when symmetric structures are placed near a high-index interface [49]. For $t=84 \mathrm{~nm}$ and varying $h$, Fig. 1(d) shows that the magnetic $\left(\alpha_{m y}^{H y}\right)$, magnetoelectric $\left(\alpha_{m y}^{E x}\right)$, and quadrupolar $\left(\alpha_{Q x z}^{E x}\right)$ responses increase monotonically while the electric dipole $\left(\alpha_{p x}^{E x}\right)$ response decreases. Thus, Figs. 1(c) and 1(d) convey a design strategy to increase $\mathbf{m}$ and $\mathbf{Q}$ by increasing the ANP tapering and height. Importantly, ANPs display $\mathbf{p}$ and $\mathbf{m}$ parallel to $\mathbf{E}$ and $\mathbf{H}$, respectively, for a normal incident plane wave. This contrasts with shallow planar structures (e.g., dolmens [49] and split rings [15]) where $\mathbf{m}$ is orthogonal to $\mathbf{H}$. Therefore, ANPs are ideally suited to realize the generalized near-field Kerker condition [55] and modify the forward-backward scattering or emission. Since the ANP magnetoelectric enhancement saturates for increased $h$, structures higher than this saturation threshold must be avoided to minimize Ohmic losses. In this spirit, the vertical lines in Figs. 1(c) and 1(d) indicate the ANP in our experiments, for which $\alpha^{S}$ is shown in Fig. 1(a).

For the experiments, we fabricated an ANP array onto a fused silica substrate by substrate conformal imprint lithography [50,56]. The ANPs have $h=150 \pm 10 \mathrm{~nm}$, $b=150 \pm 10 \mathrm{~nm}$, and $t=70 \pm 10 \mathrm{~nm}$. The lattice is squared with a constant $a=400 \pm 4 \mathrm{~nm}$. The inset in Fig. 2(a) displays an inclined-view scanning electron micrograph of the array. We spin coated on top of the array a $250 \mathrm{~nm}$ layer of polystyrene doped with the organic dye Lumogen F305 at 1 weight \% concentration. This layer thickness aids to bring out the unconventional emission properties of the ANP array, as discussed in the Supplemental Material [50]. All measurements are done at room temperature. Figure 2(a) shows the extinction, given by $1-T_{0}$, with $T_{0}$ the zeroth-order transmittance. A collimated beam from a halogen lamp impinges at normal incidence. The detector is a fiber-coupled spectrometer in the far field. The incident polarization is parallel to either of the two equivalent 

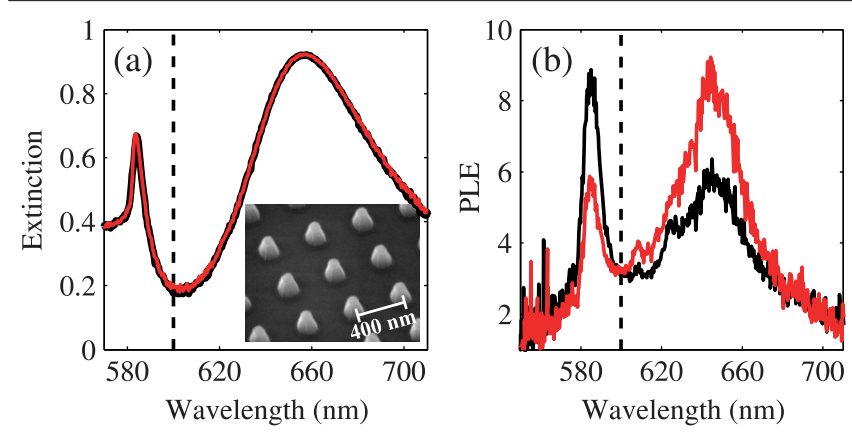

FIG. 2 (color online). (a) Measured extinction, and (b) photoluminescence enhancement (PLE) towards the top (black line) and bottom (red line) of the pyramids. The inset in (a) displays a scanning electron micrograph of the fabricated structures prior to the deposition of the luminescent layer. The dashed line in (b) indicates the Rayleigh anomaly. The PLE data are noisier at the shortest and longest wavelengths because the absolute emission intensity is weaker at these wavelengths (see the Supplemental Material [50]).

orthogonal lattice vectors. As expected based on Lorentz reciprocity, and demonstrated by the overlapping black and red lines in Fig. 2(a), the extinction is identical when the sample is illuminated from the top (the black line) or from the base of the ANPs (the red line). The broad peak near $650 \mathrm{~nm}$ in Fig. 2(a) is a localized surface plasmon resonance (LSPR) in the individual ANPs. The dip near $600 \mathrm{~nm}$ is attributed to the degenerate $( \pm 1,0)$ and $(0, \pm 1)$ RAs: diffraction orders radiating grazing to the periodicity plane. The peak at $584 \mathrm{~nm}$, with a Fano line shape [57], is a SLR associated with the LSPR-RA coupling. A refractive index of $n=1.5$, intermediate to the index of the substrate $(n=1.45)$ and of the polystyrene ( $n=1.58)$, was used to calculate the RAs. The diffracted wave acquires an intermediate effective refractive index because its energy is divided between two media [36].

Next we measure the photoluminescence towards the top and bottom of the ANPs. The pump is a $442 \mathrm{~nm}$ continuous wave laser impinging from above at $\theta_{i n}=5^{\circ}$ from the normal. The irradiance at the sample is $0.5 \mathrm{~W} / \mathrm{cm}^{2}$, which is far below saturation of the dye molecules. The detector is a fiber-coupled spectrometer with $\mathrm{NA}=4 \times 10^{-3}$. Corresponding to extinction measurements, we set in the collection path a polarization analyzer to select the emission polarized along one of the two equivalent lattice vectors, and approximately parallel (a $5^{\circ}$ offset) to the polarization of the pump. In Fig. 2(b) we plot the photoluminescence enhancement (PLE), given by the ratio of the emission from the dye layer with and without the ANPs. In both directions the PLE peaks at the LSPR and SLR wavelengths, but the magnitudes of these peaks are different. These findings demonstrate the potential of ANPs to spectrally modify the emission from planar sources otherwise emitting with equal intensities towards opposite directions. In the following, we explain this unbalanced forward-backward PLE as an emission counterpart of the Kerker condition in arrays of ANPs.
In the Supplemental Material we show through calculations of the radiative local density of optical states at the LSPR wavelength that dipoles above the ANPs in a homogeneous medium preferentially radiate towards the bottom, in agreement with our experiments [50]. Measurements show that the stratified medium does not significantly favor emission into the substrate [50]. In addition, by analyzing the coherent sum of the multipole moments constituting $\alpha^{S}$ in Fig. 1(a) (corresponding to the experimental structure), we demonstrate that $\mathbf{p}$ and $\mathbf{m}$ alone are responsible for the forward-backward asymmetry in the radiation pattern [50]. This supports the connection between our observations and the Kerker condition. Interestingly, in the measurements of Fig. 2(b) the SLR-enhanced emission is greater towards the top of the ANPs, the opposite of the LSPR case. This can be explained by a change of $\pi$ in the phase relationship between $\mathbf{p}$ and $\mathbf{m}$, as calculations suggest [50]. Next, we interpret the physics through simulations taking into account the lattice and the stratified medium.

In Figs. 3 and 4 we present 3D finite element method (COMSOL) simulations. First, by interchanging the positions of source and detector, we calculated the extinction for opposite plane wave directions. The two spectra are equal [50] (as expected based on reciprocity), with the LSPR and SLR features in agreement with the experiments. Experiments and simulations are connected by reciprocity: plane waves emitted by near-field sources correspond to near fields illuminated by plane wave sources. While reciprocity establishes this relation [16,58], it does not require the local fields to be identical for opposite illumination directions. To illustrate this point, we present the electric (E) and magnetic (H) field profiles at different planes for the LSPR in Fig. 3 and for the SLR in Fig. 4. Panels (a)-(e) in both figures correspond to top illumination, while panels (f)-(j) correspond to bottom illumination. The color scale represents the intensity enhancement with respect to the incident wave, and the arrows represent the scattered field. In panels (a)-(d),(f)-(i) in both Fig. 3 and Fig. 4, both enhancement and scattering pertain to the same field - either $\mathbf{E}$ or $\mathbf{H}$ - as indicated at the top right corner of each figure. Panels (e),(j) in both Fig. 3 and Fig. 4 show magnetic field enhancement in color and the electric scattered field as arrows to depict the magnetoelectric response.

Figures 3(a) and 3(c) show two opposed electric dipoles at $10 \mathrm{~nm}$ and $140 \mathrm{~nm}$ from the base of the ANPs. For these two dipoles to oppose each other, the scattered electric field needs to be retarded with respect to the incident field. The ANP height enables this retardation. In light of Faraday's law, from the curl of these two vector fields we expect an orthogonal magnetic dipole at an intermediate plane. Figure 3(b) shows $\mathbf{H}$ at such a plane $(50 \mathrm{~nm}$ above the base of the ANPs), where a magnetic near-field pattern is observed. Figures 3(d) and 3(e) show magnetic field enhancements localized near the lower half of the ANP. Figure 3(d) shows that the magnetic scattered field circulates the ANP without significantly coupling to adjacent ANPs. Figures 3(f)-3(j) display similar field profiles for the 

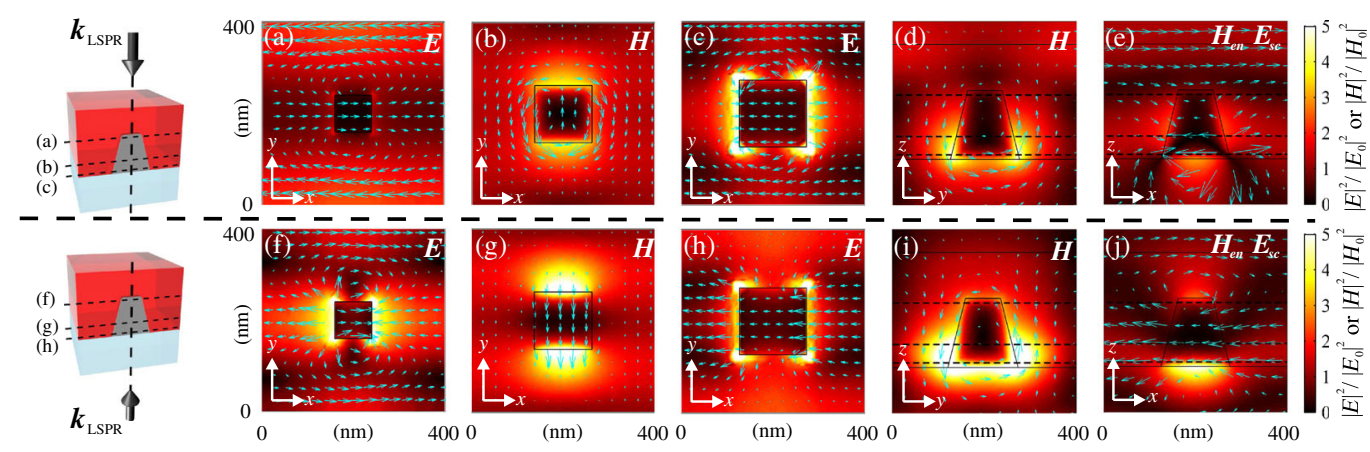

FIG. 3 (color online). Electric $(\mathbf{E})$ and magnetic $(\mathbf{H})$ fields at the localized surface plasmon resonance wavelength in simulations $(700 \mathrm{~nm})$ [50]. The color scale - equal for all plots in Figs. 3 and 4-indicates the local field intensity enhancement with respect to the incident field shown at the top right corner of each panel. The blue arrows represent the scattered field. Both colors and arrows pertain to the same field, except in (e) and (j), where we show the $\mathbf{H}$-field intensity enhancement and the scattered $\mathbf{E}$ field. Panels (a)-(e) correspond to top illumination, while panels (f)-(j) correspond to bottom illumination. The three horizontal dashed lines in panels (d),(e),(i),(j) indicate, from top to bottom, the planes at which panels (a)-(c) are evaluated for top illumination, and (f)-(h) for bottom illumination.
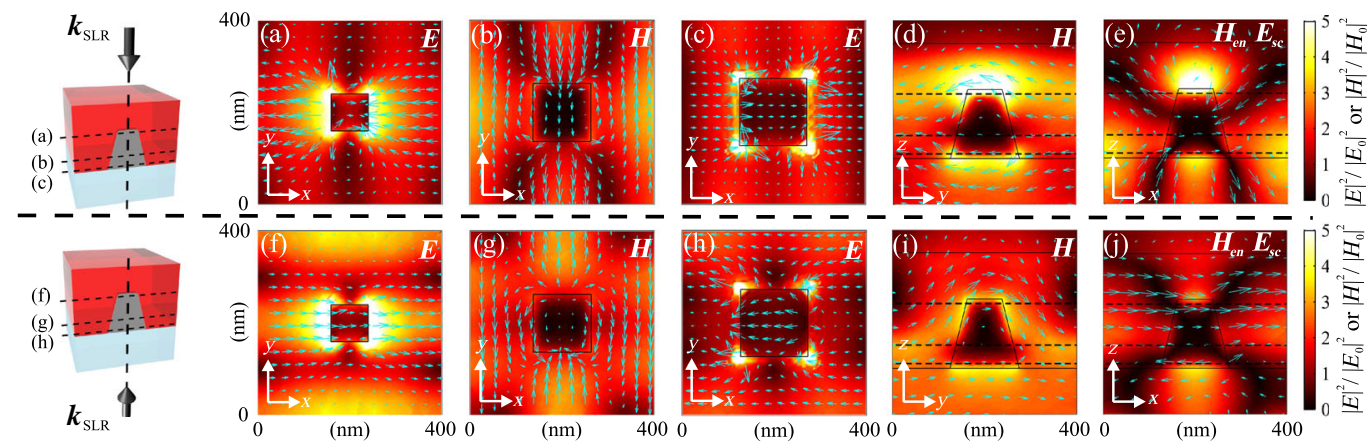

FIG. 4 (color online). Details are the same as in Fig. 3, but at the surface lattice resonance wavelength (590 $\mathrm{nm}$ ) [50].

LSPR excited from the bottom, but the field enhancements above the ANPs [Fig. 3, panels (f),(g),(i),(j)] are greater than for top illumination. By reciprocity, this implies an enhanced emission towards the bottom, in agreement with our experiments.

In Fig. 4 we present a similar analysis for the SLR. Electric and magnetic dipolelike excitations are observed at the same planes as for the LSPR. However, the SLR field enhancements are greater and the modes are collective rather than localized. SLR fields with a similar pattern but greater intensity than the associated LSPR fields also arise in structures with a dominant electric polarizability [33]. The LSPR-RA hybridization yields similar field patterns, while the large detuning makes the coupled LSPR resemble a purely localized mode and the coupled SLR resemble a long-range photonic mode. The long-range coupling at the SLR wavelength is clear in the out-of-plane magnetic [Figs. 4(d) and 4(i)] and magnetoelectric fields [Figs. 4(e) and $4(\mathrm{j})$ ], where delocalized magnetic surface waves are observed. In contrast to the LSPR, the SLR fields in the luminescent layer are greater for top illumination, in agreement with the PLE measurements. While at the LSPR p and $\mathbf{m}$ are dephased by roughly $\pi / 2$ [50] (as in a split ring [13]), at the SLR lattice interactions renormalize the polarizability [59], such that $\mathbf{p}$ and $\mathbf{m}$ are dephased by $3 \pi / 2$ and the directionality reverses. Finally, note that the opposing inplane electric dipoles for the LSPR [Figs. 3(a) and 3(c)] and the SLR [Figs. 4(a) and 4(c)] imply the existence of out-ofplane electric quadrupoles besides magnetic dipoles. While comparing the strength of these moments from simulations is difficult, calculations of $\alpha^{S}$ are insightful.

In conclusion, we demonstrated an unconventional forward versus backward luminescence enhancement from aluminum nanopyramid arrays. Underlying this effect are the enhanced magnetic and magnetoelectric response of the nanopyramids due to their tapering and height. Diffractive coupling of localized resonances in individual nanopyramids leads to collective resonances with full electromagnetic character, which constitute an unprecedented strategy for controlling light emission. The physics here explored provides a design principle for unidirectional light-emitting devices, and, by reciprocity, for boosting light absorption in unidirectionally illuminated thin-film solar cells.

This work was supported by the Netherlands Foundation for Fundamental Research on Matter (FOM) and the Netherlands Organization for Scientific Research (NWO), and it is part of an industrial partnership program between Philips and FOM. S. R. K. R., F. B. A, and T. P. S contributed equally to this Letter. 
*s.rodriguez@amolf.nl

[1] J. Pendry, A. J. Holden, D. J. Robbins, and W. J. Stewart, IEEE Trans. Microwave Theory Tech. 47, 2075 (1999).

[2] D. R. Smith, W. J. Padilla, D. C. Vier, S. C. Nemat-Nasser, and S. Schultz, Phys. Rev. Lett. 84, 4184 (2000).

[3] R. A. Shelby, D. R. Smith, and S. Schultz, Science 292, 77 (2001).

[4] V. M. Shalaev, Nat. Photonics 1, 41 (2007).

[5] J. B. Pendry, Phys. Rev. Lett. 85, 3966 (2000).

[6] N. Fang, H. Lee, C. Sun, and X. Zhang, Science 308, 534 (2005).

[7] S. Kawata, Y. Inouye, and P. Verma, Nat. Photonics 3, 388 (2009).

[8] D. Schurig, J. J. Mock, B. J. Justice, S. A. Cummer, J. B. Pendry, A. F. Starr, and D. R. Smith, Science 314, 977 (2006).

[9] T. Ergin, N. Stenger, P. Brenner, J. B. Pendry, and M. Wegener, Science 328, 337 (2010).

[10] A. Alù and N. Engheta, Phys. Rev. Lett. 102, 233901 (2009).

[11] M. Husnik, M. W. Klein, N. Feth, M. Konig, J. Niegemann, K. Busch, S. Linden, and M. Wegener, Nat. Photonics 2 , 614 (2008).

[12] C. Rockstuhl, C. Menzel, S. Mühlig, J. Petschulat, C. Helgert, C. Etrich, A. Chipouline, T. Pertsch, and F. Lederer, Phys. Rev. B 83, 245119 (2011).

[13] I. Sersic, C. Tuambilangana, T. Kampfrath, and A. F. Koenderink, Phys. Rev. B 83, 245102 (2011).

[14] M. Husnik, S. Linden, R. Diehl, J. Niegemann, K. Busch, and M. Wegener, Phys. Rev. Lett. 109, 233902 (2012).

[15] I. M. Hancu, A. G. Curto, M. Castro-López, M. Kuttge, and N. F. van Hulst, Nano Lett. 14, 166 (2014).

[16] P. Bharadwaj, B. Deutsch, and L. Novotny, Adv. Opt. Photonics 1, 438 (2009).

[17] M. Kerker, D.-S. Wang, and C. L. Giles, J. Opt. Soc. Am. 73, 765 (1983).

[18] A. García-Etxarri, R. Gómez-Medina, L. S. Froufe-Pérez, C. López, L. Chantada, F. Scheffold, J. Aizpurua, M. NietoVesperinas, and J. J. Sáenz, Opt. Express 19, 4815 (2011).

[19] S. Person, M. Jain, Z. Lapin, J. J. Sáenz, G. Wicks, and L. Novotny, Nano Lett. 13, 1806 (2013).

[20] I. Staude, A. E. Miroshnichenko, M. Decker, N. T. Fofang, S. Liu, E. Gonzales, J. Dominguez, T. S. Luk, D. N. Neshev, I. Brener, and Y. Kivshar, ACS Nano 7, 7824 (2013).

[21] A. Alu and N. Engheta, J. Nanophoton. 4, 041590 (2010).

[22] S. N. Sheikholeslami, A. García-Etxarri, and J. A. Dionne, Nano Lett. 11, 3927 (2011).

[23] S. Zou and G. C. Schatz, J. Chem. Phys. 121, 12606 (2004).

[24] E. M. Hicks, S. Zou, G. C. Schatz, K. G. Spears, R. P. Van Duyne, L. Gunnarsson, T. Rindzevicius, B. Kasemo, and M. Käll, Nano Lett. 5, 1065 (2005).

[25] F. J. García de Abajo, Rev. Mod. Phys. 79, 1267 (2007).

[26] Y. Chu, E. Schonbrun, T. Yang, and K. B. Crozier, Appl. Phys. Lett. 93, 181108 (2008).

[27] B. Auguié and W. L. Barnes, Phys. Rev. Lett. 101, 143902 (2008).

[28] V. G. Kravets, F. Schedin, and A. N. Grigorenko, Phys. Rev. Lett. 101, 087403 (2008).

[29] G. Vecchi, V. Giannini, and J. Gómez Rivas, Phys. Rev. B 80, 201401 (2009).

[30] S. R. K. Rodriguez, A. Abass, B. Maes, O. T. A. Janssen, G. Vecchi, and J. Gómez Rivas, Phys. Rev. X 1, 021019 (2011).

[31] W. Zhou and T. W. Odom, Nat. Nanotechnol. 6, 423 (2011).

[32] T. V. Teperik and A. Degiron, Phys. Rev. B 86, 245425 (2012).
[33] S. Rodriguez, M. Schaafsma, A. Berrier, and J. G. Rivas, Physica (Amsterdam) 407B, 4081 (2012).

[34] G. Weick, C. Woollacott, W. L. Barnes, O. Hess, and E. Mariani, Phys. Rev. Lett. 110, 106801 (2013).

[35] A. Abass, S. R.-K. Rodriguez, J. G. Rivas, and B. Maes, ACS Photonics 1, 61 (2014).

[36] G. Vecchi, V. Giannini, and J. Gómez Rivas, Phys. Rev. Lett. 102, 146807 (2009).

[37] G. Pellegrini, G. Mattei, and P. Mazzoldi, J. Phys. Chem. C 115, 24662 (2011).

[38] S. R. K. Rodriguez, G. Lozano, M. A. Verschuuren, R. Gomes, K. Lambert, B. D. Geyter, A. Hassinen, D. V. Thourhout, Z. Hens, and J. G. Rivas, Appl. Phys. Lett. 100, 111103 (2012).

[39] T. V. Teperik and A. Degiron, Phys. Rev. Lett. 108, 147401 (2012).

[40] G. Lozano, D. J. Louwers, S. R. K. Rodriguez, S. Murai, O. T. Jansen, M. A. Verschuuren, and J. G. Rivas, Light Sci. Appl. 2, e66 (2013).

[41] P. Offermans, M. C. Schaafsma, S. R. K. Rodriguez, Y. Zhang, M. Crego-Calama, S. H. Brongersma, and J. Gómez Rivas, ACS Nano 5, 5151 (2011).

[42] B. Špačková and J. Homola, Opt. Express 21, 27490 (2013).

[43] W. Zhou, M. Dridi, J. Y. Suh, C. H. Kim, D. T. Co, M. R. Wasielewski, G. C. Schatz, and T. W. Odom, Nat. Nanotechnol. 8, 506 (2013).

[44] S. R. K. Rodriguez, J. Feist, M. A. Verschuuren, F. J. Garcia Vidal, and J. G. Rivas, Phys. Rev. Lett. 111, 166802 (2013).

[45] S. Rodriguez and J. G. Rivas, Opt. Express 21, 27411 (2013).

[46] A. I. Väkeväinen, R. J. Moerland, H. T. Rekola, A.-P. Eskelinen, J.-P. Martikainen, D.-H. Kim, and P. Törmä, Nano Lett. 14, 1721 (2014).

[47] S. Mühlig, C. Menzel, C. Rockstuhl, and F. Lederer, Metamaterials 5, 64 (2011).

[48] F. Bernal Arango and A. F. Koenderink, New J. Phys. 15, 073023 (2013).

[49] F. B. Arango, T. Coenen, and A. F. Koenderink, ACS Photonics 1, 444 (2014).

[50] See Supplemental Material at http://link.aps.org/supplemental/ 10.1103/PhysRevLett.113.247401, which includes Refs. [51,52], for details on the units used for the superpolarizatibility tensor, the local density of optical state calculations, contributions of the electromagnetic moments to the directional radiation of aluminum nanopyramids, the full-wave simulation extinction spectrum, sample fabrication, the bare dye emission spectrum, and the influence of the dye-doped polymer layer thickness.

[51] A. M. Kern and O. J. F. Martin, J. Opt. Soc. Am. A 26, 732 (2009).

[52] B. Auguié, X. M. Bendaña, W. L. Barnes, and F. J. García de Abajo, Phys. Rev. B 82, 155447 (2010).

[53] A. Alù and N. Engheta, Phys. Rev. B 79, 235412 (2009).

[54] I. Sersic, M. A. van de Haar, F. B. Arango, and A. F. Koenderink, Phys. Rev. Lett. 108, 223903 (2012).

[55] B. Rolly, B. Stout, and N. Bonod, Opt. Express 20, 20376 (2012).

[56] M. A. Verschuuren, Ph.D. thesis, Utrecht University, 2010.

[57] M. Sarrazin, J.-P. Vigneron, and J.-M. Vigoureux, Phys. Rev. B 67, 085415 (2003).

[58] R. Carminati, M. Nieto-Vesperinas, and J.-J. Greffet, J. Opt. Soc. Am. A 15, 706 (1998).

[59] P. Lunnemann, I. Sersic, and A. F. Koenderink, Phys. Rev. B 88, 245109 (2013). 